\title{
Effect of Peridomestic Environments on Repeated Infestation by Preadult Aedes aegypti in Urban Premises in Nha Trang City, Vietnam
}

\author{
Ataru Tsuzuki,* Vu Trong Duoc, Yukiko Higa, Nguyen Thi Yen, and Masahiro Takagi \\ Department of Vector Ecology and Environment, Institute of Tropical Medicine, Nagasaki University, Nagasaki, Japan; \\ National Institute of Hygiene and Epidemiology, Hanoi, Vietnam; Center for International Collaborative Research, \\ Nagasaki University, Nagasaki, Japan
}

\begin{abstract}
To determine the effect of peridomestic environments on Aedes aegypti infestation in urban premises, we conducted two consecutive surveys in the hot-dry and cool-wet seasons. Most Ae. aegypti pupae (79\%) were recovered from premises where preadult forms (larvae and/or pupae) had been detected in both surveys. Hence, repeated infestation appears to be a useful parameter to identify premises associated with a high potential risk of dengue transmission. Multivariate analysis revealed that not only the persistent presence of containers discarded outdoors, wells, large plastic buckets, jars, and concrete toilet basins in the premises (adjusted odds ratios [aORs] = 63.3, 23.3, 22.5, 6.6, and 5.6, respectively) but also the presence of six or more residents was significantly associated with repeated infestation $(\mathrm{aOR}=6.1)$. Premises with six or more residents along with specific container types from which a large number of pupae were recovered should be targeted in dengue-control programs.
\end{abstract}

\section{INTRODUCTION}

Dengue fever and the potentially lethal shock and/or hemorrhage associated with it are rapidly increasing public health problems in over 100 countries, particularly in Southeast Asia, North and South America, and the western Pacific Islands. It is estimated that $50 \times 10^{6}$ dengue virus infections, including $50 \times 10^{4}$ cases of dengue fever with shock and/or hemorrhage, occur annually. As yet, no effective vaccine is available for dengue. To date, reduction in the population density of the vector mosquitoes, predominantly Aedes aegypti, has been the only option for controlling dengue virus transmission in the human population. ${ }^{1}$

In recent dengue-control programs, pupal/demographic surveys have been introduced as an initial step to identify epidemiologically important types of containers that should be targeted for dengue control. ${ }^{2}$ The epidemiologic importance of containers is determined on the basis of the number of pupae per container type in a pilot area(s) and on the basis of the theoretic transmission threshold that is estimated as a function of the average temperature, virus-introduction rate, and immunity of the target human population. ${ }^{3}$

Pupal/demographic surveys may ensure the development of a dengue-control strategy that focuses on container types in which the maximum number of pupae is found. ${ }^{4}$ However, this methodology does not include housing characteristics and degree of urbanization-factors that may increase the likelihood of infestation of premises by preadult forms of Ae. aegypti. Urbanization may be an important factor for the infestation of preadult forms of Ae.aegypti in an area/region, ${ }^{5,6}$ and the infestation of premises in the same area/region may vary according to the housing characteristics. In congested premises in urban areas, most water-holding containers are located indoors, and a greater number of occupants are usually associated with the presence of a larger number of waterstorage containers, owing to the high water consumption. Installation of a tap-water supply system in the premises influ-

* Address correspondence to Ataru Tsuzuki, Department of Vector Ecology and Environment, Institute of Tropical Medicine, Nagasaki University, Nagasaki 852-8523, Japan. E-mail: atarutsuzuki@hotmail .com ences the water-storage practices of the residents. Storage of rainwater that is more suitable for the breeding of $\mathrm{Ae}$. aegypti, ${ }^{7}$ may increase the chances of infestation of the premises by preadult forms of the mosquito. In addition, the difference between high- and low-rise buildings ${ }^{8,9}$; construction pattern of houses ${ }^{10}$; and the condition of the premises such as degree of shade, house, and yard conditions ${ }^{11}$ may also influence infestation. Therefore, some premises may have specific environments that increase the risk of frequent infestations by preadult forms of Ae.aegypti. The residents of these premises and their neighbors are probably more frequently exposed to $A e$. aegypti bites, and hence, are at a higher risk of dengue infection than other people. Peridomestic environmental factors related to repeated infestation by Ae. aegypti could be used to develop effective strategies for targeting specific premises in dengue-control programs.

The purpose of this study, therefore, was to determine peridomestic variables in urban areas that influence the repeated infestation of premises with Ae. aegypti. We conducted repeated house-to-house visits to inspect water-holding containers and to observe the peridomestic environment in urban premises in Nha Trang City, Vietnam.

\section{MATERIALS AND METHODS}

Nha Trang City is located in the coastal area of central Vietnam. Although the city includes 8 peri-urban communes surrounded by rice fields, most residents in the city live in 19 urban communes. We studied 2 neighborhoods in the urban communes to adjust for the effect of areas on the relationship between mosquito infestation and peridomestic environment. We selected the Bach Dang neighborhood (BD area) in Phuoc Tien commune in the central part of the city, and the eighth neighborhood (VP area) in Vinh Phuoc commune in the north of the city. These neighborhoods are situated approximately $3 \mathrm{~km}$ away from each other. In both the study areas, the premises inspected were adjacent to each other and densely populated by local residents. Most of the residents in these 2 areas were private shop owners, office/factory workers, and public officers. According to the health authorities, in 2005 the highest level of dengue cases in the city was reported from the 2 communes that included our study areas (3.3 and 4.1 cases per 1,000 persons in the Phuoc Tien and Vinh Phuoc 
communes, respectively). The city has a hot-dry season from May to October and a cool-wet season from November to April. The first survey was conducted in the hot-dry season (July 30,2006-August 5, 2006), and the second survey was conducted in the cool-wet season (December 25, 2006-January 2, 2007). All premises in the study areas were visited during both the survey periods.

Water-holding container survey. A water-holding container survey was conducted to record the presence of water-holding containers, the presence of preadult (larvae and/or pupae) Ae. aegypti in these containers, and the number of pupae. House-to-house visits were conducted by 2 teams of 3 or 4 inspectors ( 2 or 3 entomologists and 1 local community leader), generally between 8:00 AM and 6:00 PM. Locked premises were not inspected and revisited at least 2 times within 1 week after the first visit. All water-holding containers located both outdoors and indoors, including those on the top floor and in the balcony, were inspected after obtaining permission from the occupants of the premises. The container categories were based on the data obtained in former denguemosquito surveys conducted in Vietnam. ${ }^{12,13}$ Wet containers were categorized as flower vases, small or medium plastic buckets ( $\leq 99 \mathrm{~L})$, large plastic buckets $(\geq 100 \mathrm{~L})$, wells, jars, ant traps, plant saucers, concrete toilet basins (built-in concrete basins containing water for flushing excreta and for cleaning toilet floors by using small buckets or dippers; these basins are located in the toilet hut or bathroom), metal drums, concrete basins (built-in containers that hold water for drinking or cleaning dishes/clothes, generally located in the kitchen), tires (discarded tires), and containers discarded outdoors (all types of artificial containers discarded around the premises). Ceramic water tanks with levers and lids were present in toilets; however, since the water in these tanks is replenished each time the toilet is used, we did not inspect them. Small water containers with volumes of less than $5 \mathrm{~L}$ were upturned into a white plastic tray containing clean water to detect and collect immature mosquitoes. We used a mesh net to collect preadult mosquitoes from middle or large containers, except wells. Because it was difficult to access wells and to directly collect pupae from the wells by using a net, funnel traps were used for collection instead (each funnel trap was left in the well for 16-18 hours overnight). The number of pupae in the wells was estimated on the basis of the number of pupae collected in standardized funnel traps, as described in a former study. ${ }^{14}$ All of the collected larvae and pupae were kept in small plastic vials and transported to the laboratory. The number of pupae was determined, and the species was identified after the adult mosquitoes emerged. Although we did not count the number of larvae, the larval species were identified. All larvae and pupae in large water-storage containers other than wells were removed. The water in the small containers was discarded after the inspection. No container was removed for survey purposes.

Peridomestic environmental survey. Presence of a container type was defined as the persistent presence of a type of water-holding container in the premises in both the first and second surveys. The characteristics of the peridomestic environment, other than the presence of water-holding containers, were either directly observed or determined by questioning the residents during the house-to-house visits in the second container survey. We inspected the following characteristics of the peridomestic environments for each of the premises: number of stories in the main building, number of members in the household, presence of tap-water supply pipes, and whether rainwater or river water was stored in the containers.

Statistical analysis. Premises with repeated infestation were defined as premises in which at least 1 container was found to hold preadult (larvae and/or pupae) Ae. aegypti in both the first and second surveys. The association between the characteristics of the peridomestic environment and the risk of repeated infestation was assessed by calculating the odds ratios (OR) and 95\% confidence intervals (CIs). Logistic regression analysis was used to develop an explanatory model for the repeated infestation of the premises. In the multivariate analysis, we assumed that no interactions are present, and all the explanatory variables were included in the model. Diagnostic statistics pertaining to multicollinearity (varianceinflation factor) were used to evaluate collinearity between explanatory variables. We divided the premises without concrete toilet basins and jars (the most productive container types in the study) into those with and those without repeated infestation. Next, we noted the proportions of premises with $\geq 6$ residents for each of the above mentioned groups. The difference in these proportions was analyzed using the $\chi^{2}$ test, and a $P$ value of 0.05 was considered statistically significant. The data were entered into EpiData version 3.1 (EpiData Association, Odense, Denmark) and analyzed using Stata version 10.0 (Stata Corp., College Station, TX).

\section{RESULTS}

Among 120 premises in the BD area and 124 premises in the VP area, 93 and 103 residential premises (except 5 offices and 2 supplemental private schools without residential spaces, 2 small temples, and 39 residential premises that did not participate in the survey), respectively, were inspected in both the first and second surveys. All the residential premises were single-family dwellings and no multi-family apartment dwellings were found in both low- and high-rise buildings. Most of the residential buildings that were surveyed were adjacent or connected to each other, and outdoor space was absent or limited. Although the height of the buildings varied, the ground areas were almost the same in all the premises. A total of 1,438 water-holding containers were observed in the 196 premises (Table 1). The number and proportion of each container type was extremely consistent between the first and second surveys. Flower vases and small or medium sized plastic buckets were the most commonly found containers in the first $(81.0 \%$ in the BD area and $69.1 \%$ in the VP area) and second surveys $(82.3 \%$ in $B D$ area and $70.9 \%$ in the $V P$ area). Among all containers, these formed the majority that were positive for preadult (larvae and/or pupae) Ae. aegypti in the first $(52.9 \%$ in the $\mathrm{BD}$ area and $31.6 \%$ in the VP area) and second surveys $(38.6 \%$ in $\mathrm{BD}$ area and $33.8 \%$ in the VP area). However, in the first survey, these containers only accounted for $28.8 \%$ and $9.4 \%$ of the pupal production in the BD and VP areas, respectively. During the rainy season, this increased to $32.0 \%$ and $35.2 \%$. Furthermore, although the other container types were sparse, a large number of Ae. aegypti pupae were recovered from them in each survey. Jars and concrete toilet basins, which accounted for only $9 \%$ of all containers found, were the most productive container types with regard to pupae: $36 \%$ of the 1,283 Ae. aegypti pupae collected during the surveys 
TABLE 1

Distribution of water-holding containers, presence of preadult Aedes aegypti, and the number of pupae in two urban areas of NhaTrang City*

\begin{tabular}{|c|c|c|c|c|c|c|}
\hline \multirow[b]{2}{*}{ Container types } & \multicolumn{3}{|c|}{$\mathrm{BD}$ area } & \multicolumn{3}{|c|}{ VP area } \\
\hline & No. inspected & No. preadult positive & No. of pupae collected & No. inspected & No. preadult positive & No. of pupae collected \\
\hline \multicolumn{7}{|l|}{ Hot-dry season (1st survey) } \\
\hline Flower vase & 162 & 30 & 22 & 144 & 12 & 1 \\
\hline $\mathrm{S} / \mathrm{M}$ plastic bucket & 116 & 6 & 10 & 136 & 13 & 36 \\
\hline Well & 17 & 13 & 25 & 12 & 0 & 0 \\
\hline Jar & 12 & 5 & 1 & 31 & 16 & 20 \\
\hline Outdoor discarded container & 18 & 8 & 7 & 7 & 4 & 16 \\
\hline Ant trap & 4 & 2 & 0 & 12 & 10 & 9 \\
\hline Plant saucer & 1 & 1 & 0 & 2 & 2 & 0 \\
\hline Concrete toilet basin & 3 & 2 & 45 & 18 & 9 & 83 \\
\hline Metal drum & 0 & 0 & 0 & 4 & 2 & 8 \\
\hline Large plastic bucket & 8 & 0 & 0 & 27 & 7 & 58 \\
\hline Concrete basin & 2 & 1 & 1 & 8 & 1 & 99 \\
\hline Tire & 0 & 0 & 0 & 4 & 3 & 62 \\
\hline Total & 343 & 68 & 111 & 405 & 79 & 392 \\
\hline \multicolumn{7}{|l|}{ Cool-wet season (2nd survey) } \\
\hline Flower vase & 157 & 18 & 92 & 159 & 16 & 21 \\
\hline $\mathrm{S} / \mathrm{M}$ plastic bucket & 100 & 4 & 15 & 109 & 11 & 136 \\
\hline Well & 20 & 14 & 100 & 12 & 4 & 25 \\
\hline Jar & 8 & 3 & 11 & 34 & 13 & 172 \\
\hline Outdoor discarded container & 7 & 4 & 22 & 8 & 4 & 3 \\
\hline Ant trap & 5 & 5 & 10 & 11 & 7 & 0 \\
\hline Plant saucer & 4 & 4 & 26 & 0 & 0 & 0 \\
\hline Concrete toilet basin & 3 & 2 & 56 & 18 & 13 & 66 \\
\hline Metal drum & 3 & 1 & 0 & 2 & 2 & 21 \\
\hline Large plastic bucket & 3 & 1 & 1 & 16 & 7 & 0 \\
\hline Concrete bas in & 2 & 1 & 1 & 8 & 3 & 2 \\
\hline Tire & 0 & 0 & 0 & 1 & 0 & 0 \\
\hline Total & 312 & 57 & 334 & 378 & 80 & 446 \\
\hline
\end{tabular}

were recovered from these containers. The 21 Aedes albopictus pupae collected during the surveys were excluded from the analysis.

Among the 196 premises, 78 in the first survey and 77 in the second survey were infested with preadult (larvae and/or pupae) Ae. aegypti; however, only 46 premises (23.5\%) were infested in both the first and second surveys (Table 2). The premises that had Ae. aegypti-positive containers in both surveys did not account for the majority of containers with pupae (32.8\% and $30.3 \%$ in the first and second surveys, respectively); however, the majority of pupae were collected from premises with repeated infestation $(87.9 \%$ and $73.2 \%$ in the first and second surveys, respectively).

Table 3 shows the association between repeated infestation of the premises and each peridomestic variable. In the univariate analysis, the risk of repeated infestation in low-rise buildings was 4.5-fold that in high-rise buildings. The presence of 6 or more residents $(\mathrm{OR}=3.6)$ and storage of rainwater inside the premises $(\mathrm{OR}=4.5)$ also appeared to be important risk factors. The location of the premises and the total number of water-holding containers in the premises were not significantly related to the risk of repeated infestation of the premises. Only 3 premises lacked water-supply systems, and all 3 were infested repeatedly with preadult Ae. aegypti. Premises with persistent presence (presence in both the first and second surveys) of concrete toilet basins, wells, large plastic buckets, and jars had a 6.0 to 15.7 -fold higher risk of being infested in both surveys than premises that did not have these containers. However, in the multivariate analysis, after adjustment for other factors, only premises that had $\geq 6$ residents (adjusted odds ratio $[\mathrm{aOR}]=6.1$ and $\mathrm{CI}, 1.41-26.19)$ and the persistent presence of specific types of containers (aOR for containers discarded outdoors, 63.3 and CI, 2.24-1785.56; aOR for wells, 23.3 and CI, 6.62-82.24; aOR for large plastic buckets, 22.5 and CI, 2.55-197.43; aOR for jars, 6.6 and CI, 1.17-36.87; aOR for concrete toilet basins, 5.6 and CI, 1.23-25.52) appeared to be significantly associated with the risk of repeated infestation. Collinearity was not detected (variance-inflation factors, 1.031.27), and no significant interaction was observed between the explanatory variables.

TABLE 2

Infestation of preadult Aedes aegypti and pupal counts in premises

\begin{tabular}{|c|c|c|c|c|c|c|c|}
\hline \multirow[b]{2}{*}{ Premises infested by preadults* } & \multirow[b]{2}{*}{ No. of premises (\%) } & \multicolumn{3}{|c|}{ No. of containers with pupae (\%) } & \multicolumn{3}{|c|}{ No. of pupae collected (\%) } \\
\hline & & 1st survey & 2nd survey & Total & 1st survey & 2nd survey & Total \\
\hline Repeatedly (1st and 2nd) & $46(23.5)$ & $245(32.8)$ & $209(30.3)$ & $454(31.6)$ & $442(87.9)$ & $571(73.2)$ & $1013(79.0)$ \\
\hline Only 1st survey & $32(16.3)$ & $503(67.2)$ & 0 & $503(35.0)$ & $61(12.1)$ & 0 & $61(4.8)$ \\
\hline Only 2nd survey & $31(15.8)$ & 0 & $481(69.7)$ & 481 (33.4) & 0 & $209(26.8)$ & $209(16.2)$ \\
\hline None & $87(44.4)$ & 0 & 0 & 0 & 0 & 0 & 0 \\
\hline Total & $196(100)$ & $748(100)$ & $690(100)$ & $1438(100)$ & $503(100)$ & $780(100)$ & $1283(100)$ \\
\hline
\end{tabular}

$*$ Premises with at least one preadult (larvae and/or pupae) Ae. aegypti were considered as infested. 
TABLE 3

Effect of peridomestic environments on repeated infestation by preadult Aedes aegypti in the 196 premises based on logistic regression analysis*

\begin{tabular}{|c|c|c|c|c|c|c|c|}
\hline & & & & & & & sted \\
\hline & $\mathrm{n}$ & Rep & 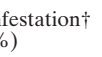 & Odds ratio & $\begin{array}{l}95 \% \text { confidential } \\
\text { interval }\end{array}$ & Odds ratio & $\begin{array}{l}95 \% \text { confidential } \\
\text { interval }\end{array}$ \\
\hline & & & & & & & \\
\hline In BD area & 93 & 20 & (21.5) & 1.0 & (ref.) & 1.0 & (ref.) \\
\hline In VP area & 103 & 26 & $(25.2)$ & 1.2 & $0.63-2.39$ & 1.4 & $0.46-4.56$ \\
\hline Peridomestic envir & & & & & & & \\
\hline Story of building & & & & & & & \\
\hline High-rise (3-4) & 39 & 3 & $(7.7)$ & 1.0 & (ref.) & 1.0 & (ref.) \\
\hline Low-rise (1-2) & 157 & 43 & (27.4) & 4.5 & $1.32-15.46$ & 1.9 & $0.32-10.81$ \\
\hline No. of residents ( $p$ & & & & & & & \\
\hline Few $(1-3)$ & 54 & 8 & $(14.8)$ & 1.0 & (ref.) & 1.0 & (ref.) \\
\hline Middle (4-5) & 90 & 18 & (20.0) & 1.4 & $0.57-3.57$ & 4.0 & $0.95-17.23$ \\
\hline Many (6-19) & 52 & 20 & (38.5) & 3.6 & $1.40-9.16$ & 6.1 & $1.41-26.19$ \\
\hline Tap water system & & & & & & & \\
\hline No & 3 & 3 & (100) & - & - & - & - \\
\hline Yes & 193 & 43 & (22.3) & - & - & - & - \\
\hline Rain water stored & & & & & & & \\
\hline No & 187 & 41 & (21.9) & 1.0 & (ref.) & 1.0 & (ref.) \\
\hline Yes & 9 & 5 & (55.6) & 4.5 & $1.14-17.33$ & 4.0 & $0.54-29.37$ \\
\hline Total no. of contai & & & & & & & \\
\hline Few $(1-4)$ & 48 & 16 & (33.3) & 1.0 & (ref.) & 1.0 & (ref.) \\
\hline Middle (5-7) & 61 & 11 & (18.0) & 0.4 & $0.18-1.06$ & 0.3 & $0.08-1.10$ \\
\hline Many (8-20) & 87 & 19 & (21.8) & 0.6 & $0.25-1.22$ & 0.4 & $0.13-1.36$ \\
\hline Presence of concr & & & & & & & \\
\hline No & 178 & 35 & (19.7) & 1.0 & (ref.) & 1.0 & (ref.) \\
\hline Yes & 18 & 11 & (61.1) & 6.4 & $2.32-17.75$ & 5.6 & $1.23-25.52$ \\
\hline Presence of well§ & & & & & & & \\
\hline No & 164 & 23 & $(14.0)$ & 1.0 & (ref.) & 1.0 & (ref.) \\
\hline Yes & 32 & 23 & (71.9) & 15.7 & $6.44-38.06$ & 23.3 & $6.62-82.24$ \\
\hline Presence of large & & & & & & & \\
\hline No & 188 & 41 & $(21.8)$ & 1.0 & (ref.) & 1.0 & (ref.) \\
\hline Yes & 8 & 5 & (62.5) & 6.0 & $1.37-26.05$ & 22.5 & $2.55-197.43$ \\
\hline Presence of jar§ & & & & & & & \\
\hline No & 185 & 39 & (21.1) & 1.0 & (ref.) & 1.0 & (ref.) \\
\hline Yes & 11 & 7 & $(63.6)$ & 6.6 & $1.82-23.52$ & 6.6 & $1.17-36.87$ \\
\hline Presence of outdo & & & & & & & \\
\hline No & 193 & 44 & $(22.8)$ & 1.0 & (ref.) & 1.0 & (ref.) \\
\hline Yes & 3 & 2 & (66.7) & 6.8 & $0.59-76.46$ & 63.3 & $2.24-1785.56$ \\
\hline Presence of concr & & & & & & & \\
\hline No & 187 & 44 & $(23.5)$ & 1.0 & (ref.) & 1.0 & (ref.) \\
\hline Yes & 9 & 2 & (22.2) & 0.9 & $0.18-4.63$ & 0.7 & $0.03-21.51$ \\
\hline Presence of flowe & & & & & & & \\
\hline No & 80 & 19 & $(23.8)$ & 1.0 & (ref.) & 1.0 & (ref.) \\
\hline Yes & 116 & 27 & (23.3) & 1.0 & $0.49-1.90$ & 1.8 & $0.59-5.43$ \\
\hline Presence of small/ & & & & & & & \\
\hline No & 88 & 26 & $(29.5)$ & 1.0 & (ref.) & 1.0 & (ref.) \\
\hline Yes & 108 & 20 & (18.5) & 0.5 & $0.27-1.05$ & 1.0 & $0.36-2.90$ \\
\hline
\end{tabular}

* BD = Bach Dang neighborhood in Phuoc Tien commune; $\mathrm{VP}=$ eighth neighborhood in Vinh Phuoc commune. $\dagger$ Premises with repeated infestation were defined as premises in which at least one container was found to hold preadult (larvae and/or pupae) Ae. aegypti in both the first and second surveys.

$\S$ Presence of a container type was defined as the persistent presence of a type of water-holding container in the premises in both the first and second surveys.

The presence of concrete toilet basins and jars in the premises was significantly associated with repeated infestation of the premises, and the highest number of pupae was found in these 2 types of containers. However, these containers were not observed in $86 \%$ (168 in 196) of the premises in either of the 2 surveys. Among the premises without toilet basins and jars, the proportion of premises with $\geq 6$ residents was significantly higher among premises with repeated infestation (48.3\% or 14 of 29$)$ than among premises without repeated infestation $(20.9 \%$ or 29 of 139$)\left(P=0.002\right.$ by $\chi^{2}$ test $)$.

\section{DISCUSSION}

Our results indicate that repeated infestation by preadult (larvae and/or pupae) Ae. aegypti in the surveys performed in 2 contrasting climatic conditions is a useful parameter to identify key premises. Collection of pupae is time sensitive, and the recovery of pupae from premises may depend on the timing of the survey in cross-sectional studies; in contrast, the presence of larvae and/or pupae in the premises is less influenced by the timing of the survey. Because temperature and rainfall are important factors influencing Ae. aegypti infestation, ${ }^{15}$ we conducted the surveys under 2 contrasting climatic conditions in the city (hot-dry and cool-wet seasons). Therefore, premises that were found to be infested with preadult (larvae and/or pupae) Ae. aegypti in both surveys are likely to be infested even at times other than the survey periods. In both surveys, most of the Ae. aegypti pupae collected in this study were isolated from premises with repeated infestation. This confirms the results of previous studies on vector population: premises with repeated infestation by preadult forms are more important from the 
viewpoint of disease transmission than premises without repeated infestation. ${ }^{16}$

Repeated infestation of premises was related to the presence of several types of containers, such as concrete toilet basins, wells, large plastic buckets, jars, and containers discarded outdoors. Besides the presence of specific types of containers, the only peridomestic variable that was associated with an increased risk of repeated infestation was the presence of a large number of residents in the premises. It remains unclear why this variable increases the risk of repeated infestation. We assumed that the presence of a greater number of residents in the premises is associated with increased water storage in a greater number of containers and therefore with increased risk of infestation by preadult forms. However, we found no association between the number of water-holding containers in the premises and repeated infestation of these premises. It is known that the egg-laying and blood-feeding behaviors of female mosquitoes are mediated through visual and olfactory cues. ${ }^{17}$ Hence, one possible reason for the increased risk of repeated infestation of premises with many residents could be the fact that in these premises, adult Ae. aegypti can feed on a greater number of hosts, and thus, a larger number of gravid mosquitoes can complete oviposition in these premises than in premises with fewer residents. Univariate analysis revealed that the presence of low-rise buildings and storage of rainwater in the premises were risk factors for repeated infestation. However, their effects were confounded by the presence of specific types of containers, which was an important risk factor for repeated infestation of the premises. This implies that the presence of low-rise buildings and storage of rainwater in the premises were not independent determinants of repeated infestation of the premises, but were observed simultaneously with the presence of containers harboring preadult Ae. aegypti in the premises.

For dengue control in urban congested premises in which most containers are located indoors, health officials should carry out house-to-house visits to detect key containers. Our results suggested that targeting premises that have $\geq 6$ residents along with key containers during house-to-house visits can effectively decrease dengue transmission. Household members who are responsible for domestic duties in premises with $\geq 6$ residents should be provided instructions regarding preventive methods pertaining to not only key container types but also to all potential Ae. aegypti breeding sites in the premises.

In Vietnam, indoor water-holding containers are common in urban premises, and the majority of preadult Ae. aegypti develop in them. ${ }^{12,18}$ Although it may be appropriate to use the characteristics of the premises alone as the criterion for the identification of productive premises in some countries, like Australia, where indoor breeding containers are not common, ${ }^{11}$ such a strategy is not useful for identifying premises with high Ae. aegypti infestation in congested urban areas in Vietnam and other countries with similar conditions. The importance of peridomestic environments in the infestation of premises with preadult Ae. aegypti should be evaluated on the basis of the housing conditions and the presence of each type of water-holding container in the premises; hence, we considered both these factors for the analysis. Installation of the tap system in the premises probably changes the residents' wateruse habits and hence has a strong impact on repeated infesta- tion of Ae. aegypti. However, because almost all premises in our study area had a tap-water supply system, we could not measure the influence of this parameter.

The results of this study show that repeated infestation of urban premises with preadult Ae.aegypti is related not only to the presence of specific types of water-holding containers but also to the presence of a large number of residents in the premises. This indicates that targeting premises with $\geq 6$ residents in addition to the specific container types from which most pupae were recovered in this study could effectively reduce the risk of dengue transmission. A follow-up survey for another year and/or the survey of more households are preferable to reach a more convincing conclusion; however, we could not conduct such a study because of resource constraints. Further studies are required to confirm our findings.

Received April 8, 2008. Accepted for publication June 28, 2009.

Acknowledgments: We express our deep gratitude to Le Viet Lo, Le Trung Nghia, Ton Nu Van Anh, Bui Cam Nhung, and Bui Thanh $\mathrm{Phu}$, in Nha Trang Pasteur Institute for field assistance. We also thank Toshihiko Sunahara for valuable suggestions.

Financial support: This work was supported by the Core University Program sponsored by the Japan Society for the Promotion of Science.

Authors' addresses: Ataru Tsuzuki and Masahiro Takagi, Department of Vector Ecology and Environment, Institute of Tropical Medicine, Nagasaki University, Nagasaki 852-8523, Japan, Tel: +81-95-8497809, Fax: +81-95-849-7812, E-mails: atarutsuzuki@hotmail.com and mstakagi@nagasaki-u.ac.jp. Vu Trong Duoc and Nguyen Thi Yen, National Institute of Hygiene and Epidemiology, Hanoi, Vietnam, E-mails:vu.duoc@gmail.com and yenanihe@yahoo.com.Yukiko Higa, Center for International Collaborative Research, Nagasaki University, Nagasaki, Japan, E-mail: yukko@nagasaki-u.ac.jp.

\section{REFERENCES}

1. Guzman MG, Kouri G, 2002. Dengue: an update. Lancet Infect Dis 2: $33-42$.

2. Barrera R, Amador M, Clark GG, 2006. Use of the pupal survey technique for measuring Aedes aegypti (Diptera: Culicidae) productivity in Puerto Rico. Am J Trop Med Hyg 74: 290-302.

3. Focks DA, Brenner RJ, Hayes J, Daniels E, 2000. Transmission thresholds for dengue in terms of Aedes aegypti pupae per person with discussion of their utility in source reduction efforts. Am J Trop Med Hyg 62: 11-18.

4. Nathan MB, Focks DA, Kroeger A, 2006. Pupal/demographic surveys to inform dengue-vector control. Ann Trop Med Parasitol 100 (Suppl 3): 1-3.

5. Tsuda Y, Suwonkerd W, Chawprom S, Prajakwong S, Takagi M, 2006. Different spatial distribution of Aedes aegypti and Aedes albopictus along an urban-rural gradient and the relating environmental factors examined in three villages in northern Thailand. J Am Mosq Control Assoc 22: 222-228.

6. Cox J, Grillet ME, Ramos OM, Amador M, Barrera R, 2007. Habitat segregation of dengue vectors along an urban environmental gradient. Am J Trop Med Hyg 76: 820-826.

7. Santana AL, Roque RA, Eiras AE, 2006. Characteristics of grass infusions as oviposition attractants to Aedes (Stegomyia) (Diptera: Culicidae). J Med Entomol 43: 214-220.

8. Liew C, Curtis CF, 2004. Horizontal and vertical dispersal of dengue vector mosquitoes, Aedes aegypti and Aedes albopictus, in Singapore. Med Vet Entomol 18: 351-360.

9. Chadee DD, 2004. Observations on the seasonal prevalence and vertical distribution patterns of oviposition by Aedes aegypti (L.) (Diptera: Culicidae) in urban high-rise apartments in Trinidad, West Indies. J Vector Ecol 29: 323-330.

10. Howell PI, Chadee DD, 2007. The influence of house construction on the indoor abundance of mosquitoes. J Vector Ecol 32: $69-74$. 
11. Tun-Lin W, Kay BH, Barnes A, 1995. The Premise Condition Index: a tool for streamlining surveys of Aedes aegypti. Am J Trop Med Hyg 53: 591-594.

12. Tran VP, Vu SN, 1999. Key breeding site of dengue vectors in Hanoi, Vietnam, 1994-1997. Dengue Bull 23: 67-72.

13. Vu SN, Nguyen TY, Tran VP, Truong UN, Le QM, Le VL, Le TN, Bektas A, Briscombe A, Aaskov JG, Ryan PA, Kay BH, 2005. Elimination of dengue by community programs using Mesocyclops (Copepoda) against Aedes aegypti in central Vietnam. Am J Trop Med Hyg 72: 67-73.

14. Kay BH, Cabral CP, Araujo DB, Ribeiro ZM, Braga PH, Sleigh AC, 1992. Evaluation of a funnel trap for collecting copepods and immature mosquitoes from wells. J Am Mosq Control Assoc 8: 372-375.
15. Focks DA, Haile DG, Daniels E, Mount GA, 1993. Dynamic life table model for Aedes aegypti (Diptera: Culicidae): analysis of the literature and model development. J Med Entomol 30: 1003-1017.

16. Chadee DD, 2004. Key premises, a guide to Aedes aegypti (Diptera: Culicidae) surveillance and control. Bull Entomol Res 94: 201-207.

17. Bentley MD, Day JF, 1989. Chemical ecology and behavioral aspects of mosquito oviposition. Annu Rev Entomol 34: 401-421.

18. Tsuzuki A, Huynh T, Tsunoda T, Luu L, Kawada H, Takagi M, 2009. Effect of existing practices on reducing Aedes aegypti preadults in key breeding containers in Ho Chi Minh City, Vietnam. Am J Trop Med Hyg 80: 752-757. 